\title{
Design Analysis of MEMS Capacitive Differential Pressure Sensor for Aircraft Altimeter
}

\author{
P. Eswaran and S. Malarvizhi
}

\begin{abstract}
In this paper, an analytical and simulation solution for Microelectromechanical systems (MEMS) based capacitive differential pressure sensor for aircraft altimeter is proposed. The principle and design of the proposed MEMS capacitive differential pressure sensor design is explained. Centre deflection and capacitive sensitivity analysis is carried out for circular, square and rectangle diaphragm membrane. Numerical analysis and simulation on deflection and capacitive sensitivity is carried out for the range of pressure from 100 mbar to1100mbar. Gold, silicon and Liquid crystal polymer (LCP) material are selected as diaphragm membrane. Analysis and simulation result shows, that gold diaphragm membrane was more suitable for aircraft altimeter applications.
\end{abstract}

Index Terms-Capacitive pressure sensor, capacitive sensitivity, differential pressure sensor MEMS, microsensor, membrane diaphragm modeling, pressure sensitivity.

\section{INTRODUCTION}

\section{A. Overview}

Micromachined MEMS piezoresistive pressure sensor finds wide applications in aerospace, medical, analytical instrumentation and commercial. MEMS pressure sensor has more advantage than conventional pressure sensor because of its low weight, low cost, reliable, smart function and occupies less space [1]. Capacitive pressure sensors provide high sensitivity to pressure, low power consumption, low noise, large dynamic range and low thermal sensitivity than piezoresistive pressure sensors [2]. Capacitive differential pressure sensor measures changes in pressure by the deflection of a conductive diaphragm due to the applied pressure. Parallel plate differential pressure sensors typically have spacer (dielectric separator) between the two electrodes and the deflection in the diaphragm due to change in pressure produces a change in capacitance [3]. The differential pressure sensor uses the ambient pressure as reference pressure and the external pressure as other source. This proposed technique reduces the package cost, eliminating the need of vacuum sealing of the diaphragm; moreover it has high sensitivity for static and dynamic pressure measurements.

\section{B. Literature Review}

In earlier work, several types of capacitance pressure

Manuscript received December 09, 2011; revised December 30, 2011.

P. Eswaran is with Department of Electronics and Communication Engineering, SRM University, Kattankulathur, Chennai, Tamilnadu,, India. 603203 (e-mail: eswaran.p@ktr.srmuniv.ac.in).

S. Malarvizhi is with Department of Electronics and Communication Engineering, SRM University, Kattankulathur, Chennai, Tamilnadu, India. 603203. sensor were analyzed and developed [1]-[13]. Most of the developed sensors were designed and fabricated to measure the absolute pressure [3], [5], [8], [10], [11]. When external pressure is applied to these sensor diaphragm membranes, the membrane deflects towards the bottom electrode and the effective capacitance increases. To increase the sensitivity, polymide materials (polymer) have been used as diaphragm membrane and capacitance change was studied over a pressure range [3]. Young et al. proposed a single crystal 3C-SiC capacitive pressure sensor for high temperature application. This sensor was demonstrated for the pressure range of $1100 \mathrm{mbar}$ to 1760 mbar [14]. G. Men, W.H. Ko presented a numerical analysis on touch mode capacitive pressure sensor with circular diaphragm [9]. D.C.Catling examined capacitive sensors that are suitable for high sensitive measurement of barometric pressure on mars (few millibars) and demonstrated capacitive prototype, with circular silicon diaphragm for the pressure range from $0 \mathrm{mbar}$ to $40 \mathrm{mbar}$ with high sensitivity at pressure $0-10 \mathrm{mbar}$ [8].

The objective of this work is to carry out an analysis on centre deflection on diaphragm membrane and capacitance sensitivity to measure a pressure below the ambient pressure (1013mbar). The pressure sensor is intended to operate in the pressure range from $100 \mathrm{mbar}$ to $1140 \mathrm{mbar}$, which is equivalent to the altitude of $50000 \mathrm{ft}$ to $-1000 \mathrm{ft}$ respectively. Deflection sensitivity and capacitive sensitivity analysis was carried out for the above range of pressure.

\section{Organization of Paper}

In section 2, the proposed capacitive differential pressure sensor principle and operation are presented. Numerical analysis on centre deflection of the diaphragm membrane and capacitance sensitivity for square, rectangle and circular diaphragm membrane are discussed in section 3 . International standard atmosphere pressure model, structure layer model of the proposed sensor, properties of diaphragms membrane materials and dimensions of sensor structure and diaphragm membrane are illustrated in section 4. Section 5 discusses the analytical and simulated results with conclusion.

\section{Proposed Differential Pressure Sensor}

The differential pressure sensor uses the pressure difference between two pressure sources. The cross-section diagram of the differential pressure capacitance pressure sensor in undeformed condition is shown in Fig. 1. The diaphragm membrane with edges built in, suspended over a sealed cavity on a silicon nitrate substrate. In this design, the cavity is sealed with local ambient pressure which is acting 
on one side of the diaphragm. The external pressure will act on the other side of the diaphragm membrane; therefore the measured pressure will be represented in gauge pressure. The element of the capacitive pressure sensor is an equivalent parallel plate capacitor with champed edges, where the diaphragm would deform responding to a differential pressure applied to two sides of the diaphragm [10]. The capacitance $\mathrm{C}=\varepsilon \mathrm{A} / \mathrm{d}$, where $\varepsilon$ is the permittivity of the dielectric of medium between the two plates, $\mathrm{A}$ is the effective area of the electrode plate and $d$ is the gap between the two plates. The upper plate of the capacitor which forms one of the electrodes deforms, due to differential pressure between the external pressure and the ambient pressure inside the chamber. The diaphragm tends to deflects outward if the external pressure is less than the ambient pressure; Cross section of differential pressure capacitive pressure sensor with deformed condition as shown in the Fig. 2.

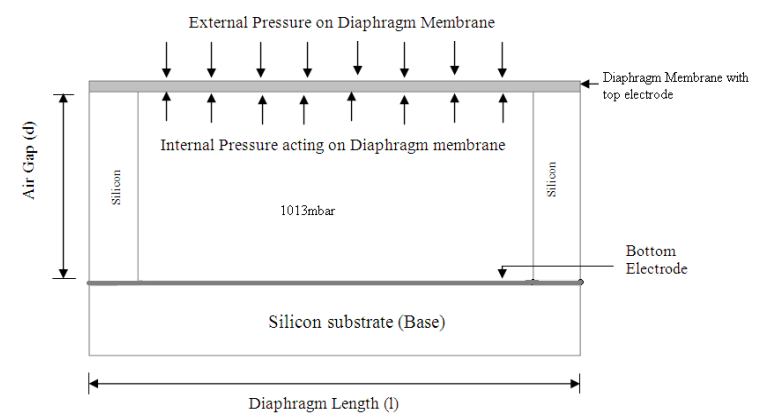

Fig. 1. Cross section of differential pressure capacitive pressure sensor

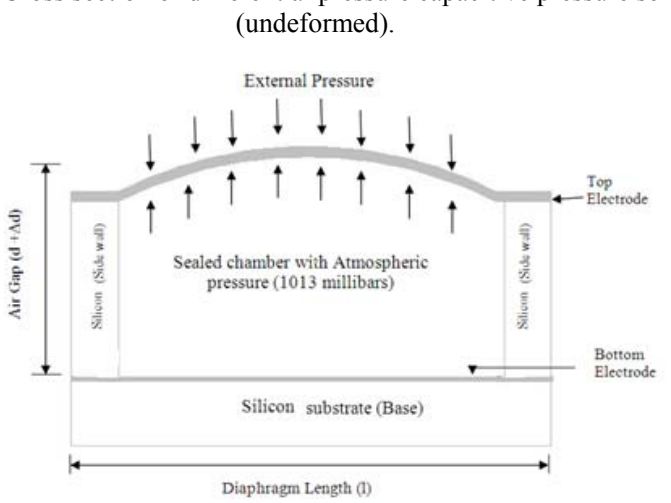

Fig. 2. Cross section of differential pressure capacitive pressure sensor (deformed).

\section{NUMERICAL ANALYSIS}

\section{A. Analysis on Deflection Sensitivity}

1) For Circular Diaphragm Membrane

The general equation for deflection of thin plate in the $x-y$ plan can be expressed [15].

$$
\frac{\partial^{4} F}{\partial x^{4}}+2 \frac{\partial^{4} F}{\partial x^{2} \partial y 2}+\frac{\partial^{4} F}{\partial y^{4}}=\left[\left(\frac{\partial^{2} w}{\partial x \partial y}\right)^{2}-\frac{\partial^{2} w}{\partial x} \cdot \frac{\partial^{2} w}{\partial y}\right]
$$

$\frac{\partial^{4} F}{\partial x^{4}}+2 \frac{\partial^{4} F}{\partial x^{2} \partial y^{2}}+\frac{\partial^{4} F}{\partial y^{4}}=\frac{h}{D}\left[\frac{P}{h}+\frac{\partial^{2} F}{\partial x^{2}} \cdot \frac{\partial^{2} F}{\partial y^{2}}+\frac{\partial^{2} F \partial^{2} w}{\partial x^{2} \partial y^{2}}-2 \frac{\partial^{2} F}{\partial x \partial y} \cdot \frac{\partial^{2} w}{\partial x \partial y}\right]$

where $D$ is flexural rigidity, $D=E h^{3} / 12\left(1-v^{2}\right), P$ is the differential pressure between two sides of a diaphragm, $w$ is deflection at point $(\mathrm{x}, \mathrm{y}), E$ is young modulus, $v$ is Poisson's ratio and $F$ is a stress function and $h$ is the thickness of the diaphragm.

The set of governing equation for circular plate under load for diaphragm membrane in non touch operation are as follows [9].

$$
\begin{gathered}
r \frac{d N_{r}}{d r}+N_{r}-N_{t} \\
D\left(\frac{d^{3} w}{d r^{3}}+\frac{1}{r} \cdot \frac{d^{2} w}{d r^{2}}\right)=N_{r} \frac{d w}{d r}+\int_{0}^{r} P E r d r \\
r \frac{d\left(N_{r}+N_{t}\right)}{d r}+\frac{E h}{2}\left(\frac{d w}{d r}\right)^{2}=0
\end{gathered}
$$

where $r$ is radius, $P$ is pressure on diaphragm, $E$ is young's modules, $h$ is thickness of plate, $v$ is Poisson's ratio, $w$ is the deflection in the direction perpendicular in radial direction, $N_{r}$ is tensile force per unit length in radial direction, $N_{t}$ is tensile force per unit length in tangential direction and $D$ is Flexural rigidity.

$$
D=\frac{E h^{3}}{12\left(1-v^{2}\right)}
$$

The centre deflection of circular diaphragm is calculated as [15].

$$
\begin{array}{r}
w_{0}=\frac{\operatorname{Pr}^{4}}{64 D} \cdot \frac{1}{1+\frac{0.488 w_{0}^{2}}{h^{2}}} \\
w_{0}=\frac{\operatorname{Pr}^{4}\left(1-v^{2}\right)}{16 E h^{3}}
\end{array}
$$

2) For Square Diaphragm Membrane

The governing differential equation for the deflection of thin plate given by [15], [16].

$$
\frac{\partial^{2} w}{\partial x^{2}}+2 \frac{\partial^{2} w}{\partial x \partial y}+\frac{\partial^{2} w}{\partial y^{2}}=\frac{P}{D}
$$

where $D$ is the flexural rigidity, by solving with boundary condition, where all sides of the sensor diaphragm are built in, that is

$$
w=0, \frac{\partial w}{\partial n}=0
$$

where $n$ is the normal vector direction along the sides of the sensor diaphragm.

The diaphragm deflection, $w(x, y)$, for a square diaphragm is given by the superposition of the deflection due to the applied pressure $w_{l}(x, y)$, which is due to the bending 
moments generated from edges restrictions, $w_{2}(x, y)$, both under simply supported edge conditions [15], [16].

Given the following variables

$$
\begin{gathered}
w(x, y)=w_{1}(x, y)+w_{2}(x, y) \\
\bar{m}=\frac{m \pi}{2}, \bar{x}=\frac{m \pi x}{L}, \bar{y}=\frac{m \pi y}{L}, \\
D=\frac{E h^{2}}{12\left(1-v^{2}\right)} \\
f(\bar{x}, \bar{y})=\cos (\bar{x})(\bar{y} \sinh (\bar{y})-\bar{m} \tanh (\bar{m}) \cosh (\bar{y}) \\
f(\bar{y}, \bar{x})=\cos (\bar{y})(\bar{y} \sinh (\bar{x})-\bar{m} \tanh (\bar{m}) \cosh (\bar{x})
\end{gathered}
$$

The deflection of diaphragm can be expressed as

$$
\begin{aligned}
& w_{1}(x, y)=\frac{4 P L^{4}}{\pi^{5} D} \sum_{m=1,3,5}^{\infty} \frac{(-1)^{\frac{m-1}{2}}}{m^{5}} \cos (\bar{x}) \times \\
& \left(1-\frac{\bar{m} \tanh (\bar{m})+2}{2 \cos (\bar{x})} \cosh (\bar{y})+\frac{\bar{y}}{2 \cosh (\bar{m})} \sinh (\bar{y})\right) \\
& w_{2}(x, y)=\frac{-2 P L^{4}}{\pi^{5} D} \sum_{m=1,3,5}^{\infty} e_{m} \frac{(-1)^{\frac{m-1}{2}}}{m^{2} \cosh (\bar{m})} \times \\
& (f(\bar{x}, \bar{y})+f(\bar{y}, \bar{x}))
\end{aligned}
$$

where $L, E, \mathrm{~h}, v$ is diaphragm length, Young's modulus, diaphragm thickness, and Poisson's ratio respectively. Coefficients $e_{m}$ are derived from the same procedure of Ref. [15] and we can get

$\mathrm{e}_{1}=-0.372, \quad \mathrm{e}_{3}=-0.379, \quad \mathrm{e}_{5}=-0.0175, \quad \mathrm{e}_{7}=-0.0082, \quad \mathrm{e}_{9}$ $=-0.00428, \quad \mathrm{e}_{11}=0.00243, \mathrm{e}_{13}=0.00147, \mathrm{e}_{15}=0.0009, \mathrm{e}_{17}=$ 0.0006 , and $\mathrm{e}_{19}=0.0004$

Then, the bending moment $M_{x}$ and $M_{y}$ are calculated by means of

$$
\begin{aligned}
& M_{x}=-D\left(\frac{\partial^{2} w}{\partial x^{2}}+v \frac{\partial^{2} w}{\partial y^{2}}\right) \\
& M_{y}=-D\left(\frac{\partial^{2} w}{\partial y^{2}}+v \frac{\partial^{2} w}{\partial x^{2}}\right)
\end{aligned}
$$

The diaphragm plates are stress magnifying device. Since the diaphragm plate is sensitive to stress, it is necessary to evaluate the stress distribution of a plate. The stress can be calculated by

$$
\sigma_{x}=6 \frac{M_{x}}{h^{2}}
$$

$$
\sigma_{y}=6 \frac{M_{y}}{h^{2}}
$$

The maximum deflection of the diaphragm is the centre of diaphragm. We get the maximum deflection by superposition, that is $\quad w_{\max }=w_{1}+w_{2}$. We can get $w_{1}(0,0)=0.00406 P L^{4} / D \quad, \quad w_{2}=-0.00280 P L^{4} / D \quad$, $w_{\max }=0.00126 P L^{4} / D$. If the $E h^{3} / 12\left(1-v^{2}\right)$ is used instead of $\mathrm{D}$, then the maximum deflection became, Obviously the maximum diaphragm deflection is linearly proportional to applied pressure.

$$
w_{\max }=0.01512\left(1-v^{2}\right) \frac{P L^{4}}{E h^{3}}
$$

3) For Rectangular Diaphragm Membrane

The diaphragm centre deflection $w(x, y)$ for rectangle diaphragm of Ref. [15] is given by

$$
\begin{gathered}
w_{\max }=\alpha \frac{P b^{4}}{E h^{3}} \\
\alpha=1.26 \times 10^{-3} \times 12\left(1-v^{2}\right)
\end{gathered}
$$

TABLE I: COEFFICIENT A FOR DIFFERENT RATIO OF B/A DIMENSION OF RECTANGULAR MEMBRANE DiAPHRAGM [15].

\begin{tabular}{|c|c|c|c|c|c|c|}
\hline \multicolumn{7}{|c|}{ RECTANGULAR MEMBRANE DIAPHRAGM } \\
\hline$b / a$ & 1.0 & 1.3 & 1.4 & 1.5 & 1.6 & $\propto$ \\
\hline$w_{\max }(x=0, y=0)$ & $0.00126 \frac{P a^{4}}{D}$ & $0.00191 \frac{P a^{4}}{D}$ & $0.00207 \frac{P a^{4}}{D}$ & $0.00220 \frac{P a^{4}}{D}$ & $0.00230 \frac{P a^{4}}{D}$ & $0.00260 \frac{P a^{4}}{D}$ \\
\hline
\end{tabular}

where $D, P, \alpha, w_{\max }(x, y)$ is flexural rigidity, pressure in $\mathrm{MPa}$, coefficient of the rectangle thin plate and centre deflection. Where $b / a=1$ for square diaphragm, where $a$ and $b$ are the width and length of the rectangle plate.

\section{B. Analysis on Capacitance Sensitivity Measurement}

1) For Circular Diaphragm Membrane

The capacitive sensitivity for the pressure can be calculated as [13].

$$
\begin{gathered}
\Delta C=C-C_{0}=\iint_{A} \frac{\varepsilon d A}{d-w(r)}-\frac{\varepsilon A}{d} \\
C=\iint \frac{\varepsilon}{d-w(r)} r d r d \theta \\
w(r)=\frac{P A^{4}}{64 D}\left[1-\left(\frac{r}{A}\right)^{2}\right]^{2}
\end{gathered}
$$

where $C$ is the capacitance corresponds to differential pressure $P, C_{o}$ is the capacitance at zero differential pressure. $\varepsilon_{r}$ is the relative permittivity of the dielectric wall separates the top and bottom electrodes, $A$ is the area of the plate, and $w(r)$ is the centre deflection corresponds to the differential pressure. $d, r, D$ is distance between the electrode, radius of 
the diaphragm and flexural rigidity respectively.

For circular diaphragm sensor the effective capacitance, the fixed capacitance constituted by Si dielectric and variable capacitance with air dielectric is given in equation (27) to (32).

$$
\begin{gathered}
C_{\text {eff }}=C_{\text {Dielectric }}+C_{\text {Air }} \\
C_{\text {Dielectric }}=\frac{A_{d} \varepsilon_{r} \varepsilon_{0}}{D_{d}} \\
C_{\text {Air }}=\frac{\varepsilon_{r} A_{a}}{D_{d}+\Delta d} \\
A_{d}=A_{T}-A_{a} \\
A_{T}=\pi r_{0}^{2} \\
A_{a}=\pi r_{i}^{2}
\end{gathered}
$$

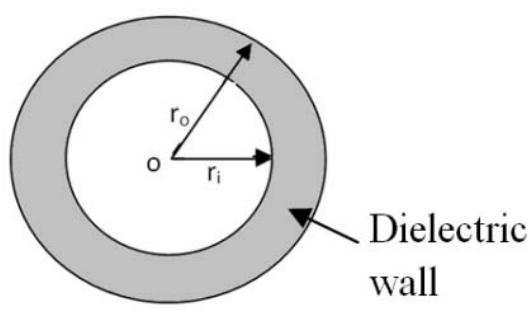

Fig. 3. Top view of Circular dimension sensor spacer structure.

Fig. 3 Shows a lateral sectional view of circular dimension sensor dielectric spacer structure. From the above equations where $A_{T}$ is the total area of the circular diaphragm; $A_{A}$ is the area of the circular diaphragm separated by air as dielectric; $A_{d}$ is the area of the circular diaphragm under dielectric, $r_{i}$ is the inner radius of diaphragm with air gap, $r_{0}$ is the outer radius of diaphragm.

The proposed capacitance pressure sensor was designed with two electrodes separated by a dielectric medium, a hollow Si substrate as dielectric with wall thickness $D_{t}$. The lateral sectional view of dielectric wall is shown in Fig. 3. Therefore the effective capacitance $C_{\text {eff }}$ of this sensor can be calculated by two sections, one with a fixed capacitance $C_{\text {Dielectric }}$ obtained with fixed dielectric wall and the other with capacitance $C_{A i r}$ due to the differential pressure acting on the diaphragm membrane area with air as dielectric. It is given in the equation (27).

$$
C_{\text {eff }}=C_{\text {Dielectric }}+C_{\text {Air }}
$$

2) For Square and Rectangle Diaphragm Membrane The effective capacitance of the sensor is given by the sum of the capacitance constituted by $\mathrm{C}_{\text {Dielectric }}$ and $\mathrm{C}_{\text {Air was }}$ given in equation (34) and (35) respectively.

$$
\begin{gathered}
C_{\text {Dielectric }}=\frac{2 \varepsilon_{r} \varepsilon_{0}\left(l \times b_{a}\right) D_{t}}{D_{d}} \\
C_{\text {Air }}=\frac{\varepsilon_{r} \varepsilon_{0}\left(l \times D_{t}\right) b_{a}}{D_{d}+\Delta d}
\end{gathered}
$$

where $l$ is the length of the diaphragm wall, $b_{a}$ is the inner width of the (cavity), $D_{t}$ is the wall thickness of the diaphragm, $D_{d}$ is the height of dielectric separating the electrodes, $\varepsilon_{0}$ is the permittivity of $8.854 \times 10^{-12} \mathrm{f} / \mathrm{m}$, $\mathcal{E}_{r}$ is the dielectric permittivity of Si and $D_{d}$ is the change in centre displacement of diaphragm with reference zero differential pressure acting on diaphragm.

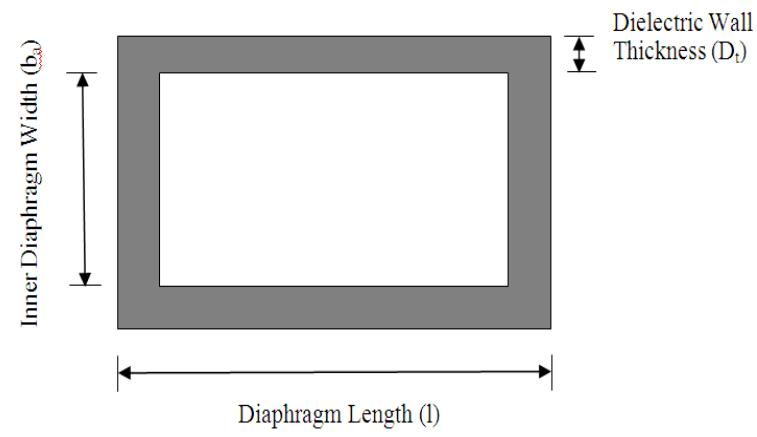

Fig. 4. Top view of rectangle dimension sensor spacer structure.

\section{Simulation Model}

\section{A. Proposed Structure of Capacitive Differential Pressure} Sensor

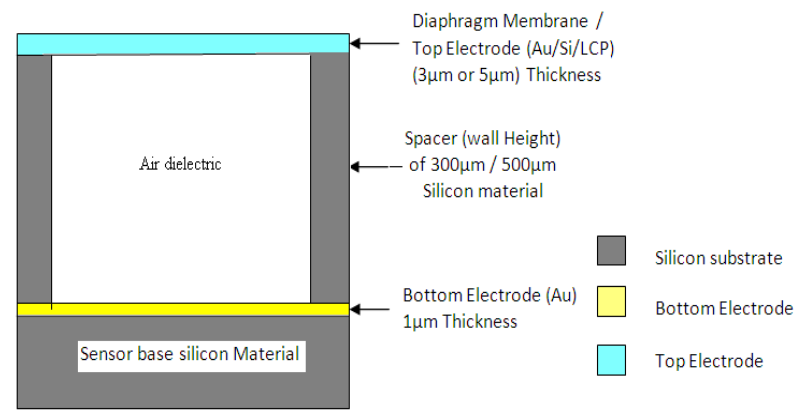

Fig. 5. Cross section Sideview of sensor structure.

The capacitive differential pressure sensor made up of four layers. Fig. 5. Shows the sectional view of sensor structure layers. Silicon material ( $\mathrm{Si}$ ) is used as a constraint base layer at with thickness of $100 \mu \mathrm{m}$, a thin film of gold material deposited over Si base layer by CVD process (chemical vapor deposition) with a thickness of $1 \mu \mathrm{m}$, which form one of the electrode of capacitive pressure sensor. A Si spacer side wall serves as two purpose, used to separate the top and bottom electrode of the capacitive sensor and it act as a fixed solid dielectric medium with a permittivity of $\varepsilon_{\mathrm{r}}=11.6$.

The cavity is sealed with a diaphragm membrane with the 
thickness of $3 \mu \mathrm{m}$ and $5 \mu \mathrm{m}$ (same value is used in analysis) with atmospheric pressure inside. The top layer works in two ways, as diaphragm is subjected to differential pressure and the diaphragm itself acts as one of the electrode parallel plate of capacitive pressure sensor.

\section{B. International Standard Atmosphere (ISA)}

Atmospheric pressure variation equivalent to the altitude of $-1000 \mathrm{ft}$ to $50,000 \mathrm{ft}$ used in this analysis. The effect of temperature on capacitive pressure sensor is negligible [2], hence this effect was not considered in this analysis. Fig. 6. Shows the relationship between the altitudes in feet's with pressure in millibar [17].

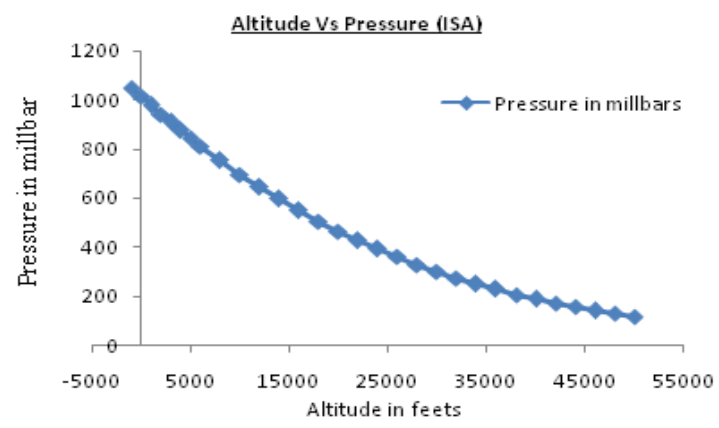

Fig. 6. Representation of international Standard atmosphere with relationship between altitude and pressure variation.

\section{Diaphragm Membrane Materials}

In this analysis and simulation gold, Liquid crystal polymer (LCP) and silicon materials are used as the diaphragm membrane material. The material parameters used are illustrated in TABLE II.

TABLE II: DiAPHRAGM MEMBRANE MATERIAL PARAMETER.

\begin{tabular}{|c|c|c|c|}
\hline Material Properties & Silicon & Gold & LCP \\
\hline Young's Modulus E $(\mathrm{GPa})$ & $130-187$ & 78 & $7-22$ \\
\hline Density $\left(\mathrm{Kg} / \mathrm{M}^{3}\right)$ & 2300 & 19300 & 1400 \\
\hline Poisson ratio $(v)$ & 0.28 & 0.42 & 0.4 \\
\hline Dielectric Constant $\left(\varepsilon_{\mathrm{r}}\right)$ & 11.6 & $2.3 \times 10-8$ & 2.8 \\
\hline
\end{tabular}

\section{Sensor Structure and Dimensions.}

Square, rectangular and circular form structures were choosen for analysis. The diaphragm membrane of dimension range from $500 \mu \mathrm{m} \times 500 \mu \mathrm{m}$ to $1000 \mu \mathrm{m} \times 1000 \mu \mathrm{m}$ is used most commercial designs [6]. In this analysis the dimension for square membrane are $750 \mu \mathrm{m} \times 750 \mu \mathrm{m}$ with $3 \mu \mathrm{m}$ and $5 \mu \mathrm{m}$ membrane thickness. For rectangle diaphragm membrane the dimension of $1000 \mu \mathrm{m} \times 750 \mu \mathrm{m}, 150 \mu \mathrm{m} \times$ $1000 \mu \mathrm{m}$ with $3 \mu \mathrm{m}$ and $5 \mu \mathrm{m}$ membrane thickness and for circular diaphragm membrane with $500 \mu \mathrm{m}, 1000 \mu \mathrm{m}$ diameter with $3 \mu \mathrm{m}$ and $5 \mu \mathrm{m}$ membrane thickness are used.

\section{RESULTS AND DiSCUSSION}

Numerical analysis is carried for different dimension, thickness of diaphragm membrane material. For the same numerical values modeling and simulation was carried out with Intellisuit v.8.51 in the thermo electromechanical analysis (TEM) module.

\section{A. Displacement Sensitivity}

The displacement sensitivity is the rate of change of centre displacement per unit change in pressure expressed in $\mathrm{nm} / \mathrm{mbar}$. The centre displacement sensitivity for circular, square and rectangular diaphragm membrane was analyzed and simulated for a range of pressure from 100mbar to $1100 \mathrm{mbar}$ (Pressure range of aircraft flying altitude). Fig. 7. shows the center deflection sensitivity with differential pressure acting on circular diaphragm membrane. Gold and silicon diaphragm membrane material are selected for analysis. The gold diaphragm with dimensions of $500 \mu \mathrm{m}$ diameter with a diaphragm thickness of $5 \mu \mathrm{m}$ and for silicon with diaphragm dimension of $1000 \mu \mathrm{m}$ diameter with diaphragm thickness of $5 \mu \mathrm{m}$ was selected. The centre displacement sensitivity for gold diaphragm was found to be $28 \mathrm{~nm} / \mathrm{mbar}$ and silicon diaphragm membrane shows the displacement sensitivity of $8 \mathrm{~nm} / \mathrm{mbar}$ and $247 \mathrm{~nm} / \mathrm{mbar}$ for $500 \mu \mathrm{m}$ and $1000 \mu \mathrm{m}$ diameter respectively.

The displacement sensitivity with differential pressure on a square diaphragm membrane of gold and LCP materials are shown in the Fig. 8. The gold membrane of dimension $750 \mu \mathrm{m} \times 750 \mu \mathrm{m}$ with diaphragm thickness of $3 \mu \mathrm{m}$ shows the sensitivity of $113 \mathrm{~nm} / \mathrm{mbar}$. It gives linear displacement variation with high sensitivity. The LCP membrane shows the sensitivity of $90 \mathrm{~nm} / \mathrm{mbar}$.

Fig. 9 Illustrate the centre displacement of rectangular diaphragm membrane of silicon and gold material. The dimension of $1000 \mu \mathrm{m} \times 750 \mu \mathrm{m}$ with diaphragm thickness of $3 \mu \mathrm{m}$ shows the displacement sensitivity of $181 \mathrm{~nm} / \mathrm{mbar}$ and $160 \mathrm{~nm} / \mathrm{mbar}$ for gold and LCP membrane diaphragm respectively. Silicon diaphragm membrane with $1500 \mu \mathrm{m} x$ $1000 \mu \mathrm{m}$ shows the displacement sensitivity of $169 \mathrm{~nm} / \mathrm{mbar}$.

Comparing the displacement sensitivities of square, rectangle and circular diaphragm membrane, the circular diaphragm membrane with gold membrane shows very high sensitivity. Square diaphragm shows more sensitivity then the rectangular diaphragm membranes. The sensitivity of the LCP material with square diaphragm also close to gold diaphragm membrane material, but it is not suitable for this application due to non availability of standard manufacturing technique for fabricating very thin diaphragm.

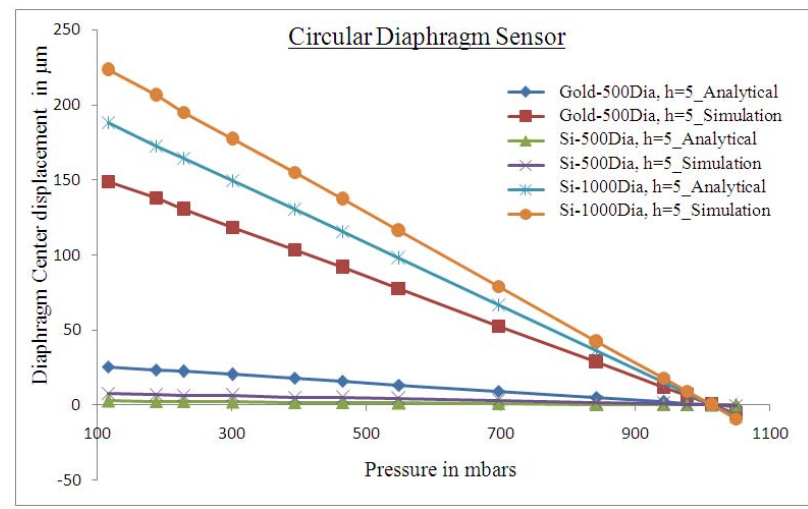

Fig. 7. Center deflection sensitivity verses differential pressure on Circular diaphragm membrane. 


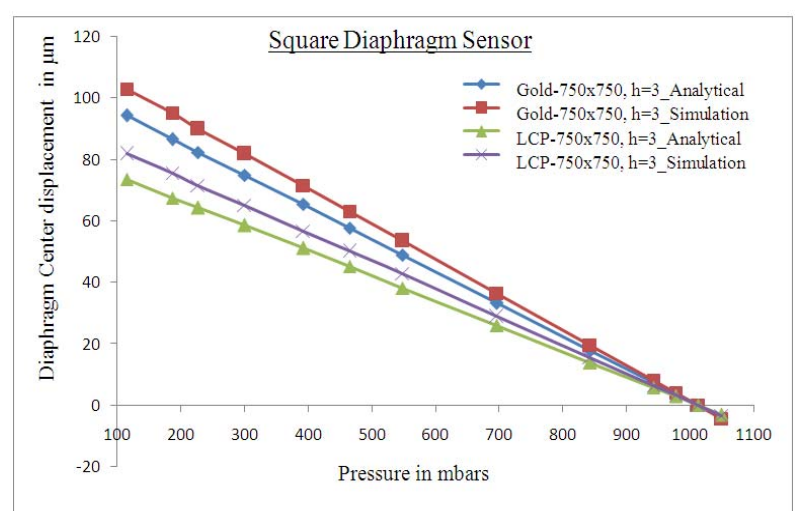

Fig. 8. Center deflection sensitivity verses differential pressure on square diaphragm membrane.

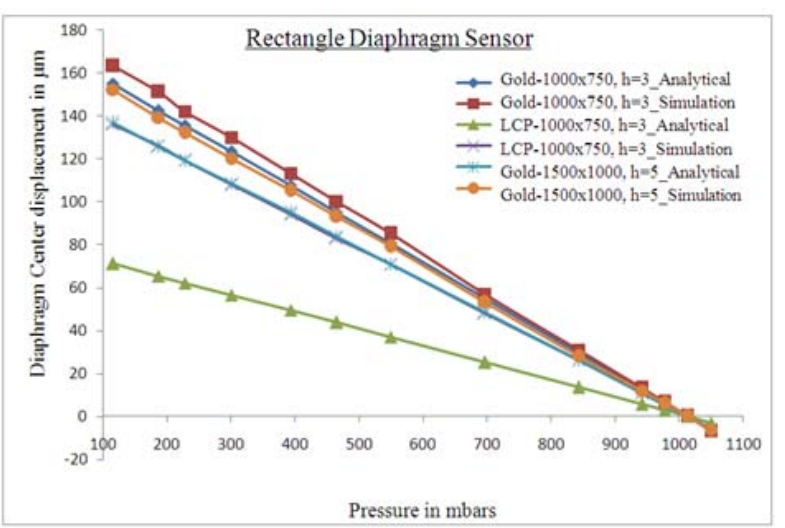

Fig. 9. Center deflection sensitivity verses differential pressure on rectangle diaphragm membrane.

\section{B. Capacitive Sensitivity}

Fig. 10 Shows the capacitance variation as a function of differential pressure on circular diaphragm membrane. The capacitive sensitivity of $500 \mu \mathrm{m}$ circular diaphragm with gold and silicon membrane material shows $1.15 \mathrm{aF} / \mathrm{mbar}$ and $0.03 \mathrm{aF} / \mathrm{mbar}$ respectively, $1000 \mu \mathrm{m}$ circular diaphragm shows the sensitivity of $5.6 \mathrm{aF} / \mathrm{mbar}$.

The capacitive sensitivity for the square diaphragm with gold and LCP membrane was found as $2.033 \mathrm{aF} / \mathrm{mbar}$ and $1.662 \mathrm{aF} / \mathrm{mbar}$ respectively as shown in the Fig. 11. For rectangular membrane diaphragm the gold and silicon membrane with dimensions $1000 \mu \mathrm{m} \times 750 \mu \mathrm{m}$ shows the sensitivity of $4 \mathrm{aF} / \mathrm{mbar}$ and $3.6 \mathrm{aF} / \mathrm{mbar}$ respectively. $1500 \mu \mathrm{m} \times 1000 \mu \mathrm{m}$ gold diaphragm membrane shows sensitivity of $8.3 \mathrm{afF} / \mathrm{mbar}$ as shown in Fig. 12.

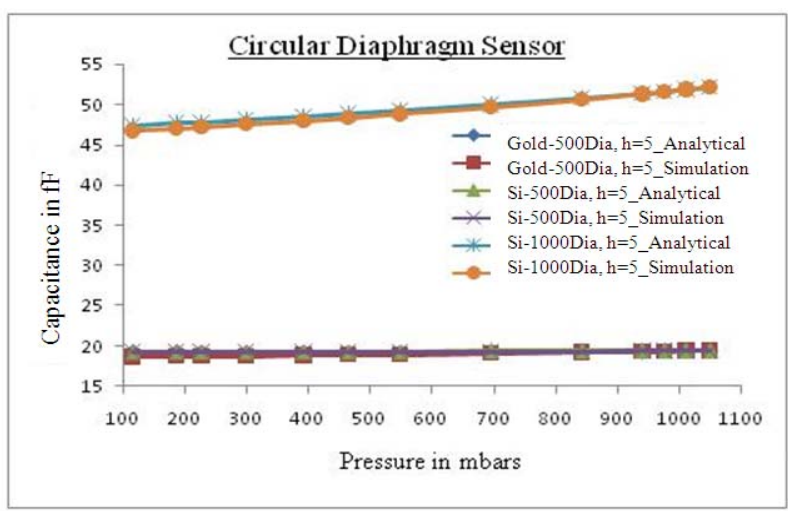

Fig. 10. Capacitance variation as a function of differential pressure on circular diaphragm membrane.

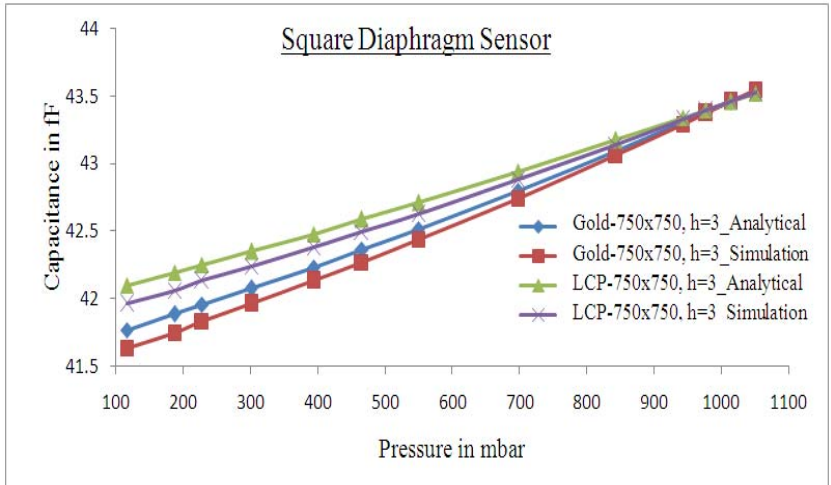

Fig. 11. Capacitance variation as a function of differential pressure on square diaphragm membrane.

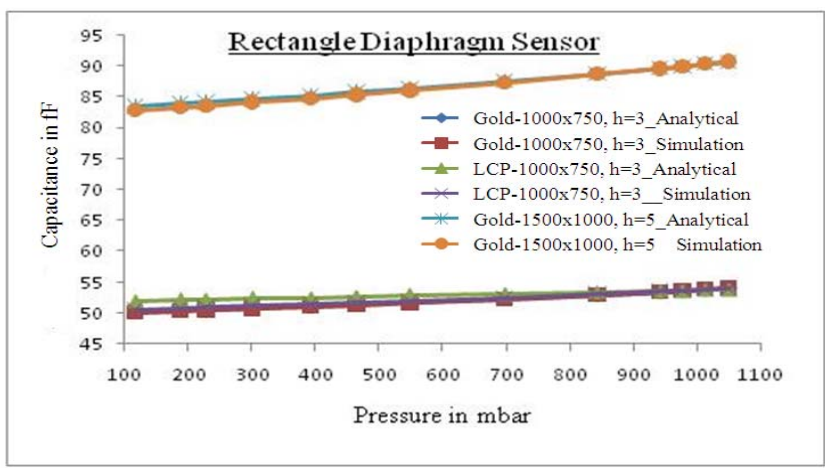

Fig. 12. Capacitance variation as a function of differential pressure on rectangle diaphragm membrane.

It is expected that the sensitivity should be uniform over the entire range of pressure. In this diaphragm of gold material with dimension $750 \mu \mathrm{m} \times 750 \mu \mathrm{m}$ with $3 \mu \mathrm{m}$ diaphragm thickness shows a less sensitivity deviation over the range of working pressure. The circular diaphragm with silicon membrane shows very high sensitivity variation.

\section{CONCLUSION}

Capacitive differential pressure sensor for aircraft altimeter applications was proposed. Structure design model and simulation was carried out. Obtained simulation results are compared with the values obtained numerically. The gold diaphragm membrane shows high sensitivity on centre deflection and capacitance, on comparing with silicon and LCP membrane. It has excellent linear variation of deflection over the working range of pressure. From this analysis all the dimensions of diaphragm membranes shows higher deflection sensitivity at lower altitude when compare with higher altitude, this is due to the large pressure variation at lower altitude, than with higher altitude.

\section{ACKNOWLEDGEMENTS}

The Author would like to acknowledge NPMASS program at SRM University for providing the facility to carry out this work.

\section{REFERENCES}

[1] L. Lin and W. Yun, "MEMS Pressure Sensors for Aerospace Applications," Proceeding IEEE Aerospace Conference, vol. 1, Colorado, 21-28, pp. 429- 436, March 1998.

[2] Y. Lee and K. D. Wise, "A Batch-Fabricated Silicon Capacitive Pressure Transducer with Low Temperature Sensitivity," IEEE 
Transactions on Electron Devices, vol. Ed-29, no.1, pp. 42-48, Jan. 1982.

[3] J. Han and et.al, "Smooth Contact Mode Capacitive Pressure Sensor with Polyimide Diaphragm," Proceeding of IEEE conference on Sensors, Atlanta, 28-31, pp. 1468-1471, Oct. 2007.

[4] J. Han and M. A. Shannon, "Smooth Contact Capacitive Pressure Sensors in Touch- and Peeling-Mode Operation," IEEE Sensors Journal, vol. 9, no. 3, pp. 199-209, March 2009.

[5] F. He, Q. -A. Huang and M. Qin, "A silicon directly bonded capacitive absolute pressure sensor," Sensors and Actuators A 135, pp. 507-514, 2007.

[6] S. -C. Gong, "Effects of pressure sensor dimensions on process window of membrane thickness," Sensors and Actuators A 112, pp. 286-290, 2004.

[7] D. J. Young, J. Du, C. A. Zorman, and W. H. Ko, "High-Temperature Single-Crystal 3C-SiC Capacitive Pressure Sensor," IEEE Sensors Journal, vol. 4, no. 4, pp. 464-469, Aug. 2004.

[8] D. C. Catling, "High sensitive capacitive pressure sensor for measuring medium-vacuum gas pressure," Sensor and Actuator A 64, pp. 157-164, 1998.

[9] G. Meng and W. H. Ko, "Modelling of circular diaphragm and spreadsheet solution programming for touch mode capacitive sensors," Sensors and Actuators A vol.75, pp. 45-52, 1999.

[10] Wen H. Ko and Qiang Wang, "Touch mode capacitive pressure sensors," Sensors and Actuators A, vol.75, pp. 242-251, 1999.

[11] Q. Wang and W. H. Ko, "Modelling of touch mode capacitive sensors and diaphragms," Sensors and Actuators A vol.75, pp. 230-241, 1999.

[12] L. Kaabi, J. Saklya, and M. Saafib, "A low pressure meter based on a capacitive microsensor," Proceedings of the JMSM 2008 Conference, vol. 2, Issue 3, pp. 1495-1503, Nov. 2009.
[13] F. He, Q. -A. Huang, and M. Qin, "A silicon directly bonded capacitive absolute pressure sensor," Sensors and Actuators A vol. 135, pp. 507-514, 2007.

[14] D. J. Young, J. Du, C. A. Zorman, and W. H. Ko, "High-Temperature Single-Crystal 3C-SiC Capacitive Pressure Sensor," IEEE Sensors Journal, Vol. 4, No. 4, pp. 464-469, Aug. 2004.

[15] S. Timoshenko and S. Woinowsky-Krieger, "Theory of Plates and Shells," New York, McGraw-Hill., 1959.

[16] S. -C. Gong and C. Lee, "Analytical Solutions of Sensitivity for Pressure Microsensor," IEEE Sensors Journal, Vol. 1, No. 4, pp. 340-344, Dec. 2001.

[17] US Standard atmosphere, 1976, NASA-TM-X-74335.

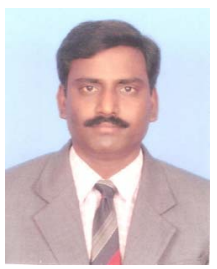

P. Eswaran was born in Chennai, India in 1974. He received his Bachelor degree in Electronics and Telecommunication Engineering from the Institute of Engineers (India) in 2000 and Masters with the specialization in Mechatronics from Anna University, Tamilnadu, India. Currently he is working as Assistant professor in the department of Electronics and Communication Engineering at SRM University, Chennai, India also pursuing his $\mathrm{PhD}$ degree in the area of MEMS in the same university.

S. Malarvizhi was Professor in the department of Electronics and Communication Engineering at SRM University, Chennai, India. Her area of interest is MEMS, VLSI, WSN, and MIMO Communication. 